ÉGYPTE monde arabe

\section{Égypte/Monde arabe}

18-19| 1994

L'éducation en Égypte

\title{
Les enseignants égyptiens. Un siècle de syndicalisme
}

\section{Muhammad Abu-I-As'ad}

Traducteur : Iman Farag

\section{CpenEdition \\ Journals}

Édition électronique

URL : https://journals.openedition.org/ema/77

DOI : $10.4000 /$ ema.77

ISSN : 2090-7273

Éditeur

CEDEJ - Centre d'études et de documentation économiques juridiques et sociales

Édition imprimée

Date de publication : 30 septembre 1994

Pagination : $39-53$

ISSN : 1110-5097

Référence électronique

Muhammad Abu-I-As'ad, "Les enseignants égyptiens. Un siècle de syndicalisme », Égypte/Monde arabe [En ligne], 18-19 | 1994, mis en ligne le 08 juillet 2008, consulté le 07 juillet 2022. URL : http:// journals.openedition.org/ema/77 ; DOI : https://doi.org/10.4000/ema.77

Ce document a été généré automatiquement le 7 juillet 2022

Tous droits réservés 


\title{
Les enseignants égyptiens. Un siècle de syndicalisme
}

\author{
Muhammad Abu-I-As'ad
}

Traduction : Iman Farag

1 L'histoire de la mobilisation syndicale des enseignants égyptiens peut se découper en quatre étapes : la première, de 1891 à la révolution de 1919, est celle de l'émergence du mouvement. La seconde, jusqu'en 1950, voit naître au sein de la profession une grande diversité d'expressions syndicales et corporatistes. Dans une troisième étape, de 1951 (antérieurement à la révolution de juillet 1952) à 1981, c'est la figure de l'organisation syndicale unique qui domine, avec la mise en place de l'Ordre des professions enseignantes. Celui-ci continue à occuper la totalité du paysage durant la dernière période, inaugurée en 1981 par la «quatrième République » du président Moubarak et caractérisée par une multiplicité des formes de mobilisation au sein de la base enseignante, parfois amenée à se prononcer contre les options de l'Ordre.

\section{L'émergence d'une mobilisation collective : 1891-1919}

2 La première mobilisation collective est celle qui, en 1891, réunit les instituteurs fraîchement diplômés de l'École normale Tawfiqiyya (Madrasat al-mu'allimîn altawfiqiyya). L'Association d'entente (Gam'iyyat at-i'tilâf) a pour but de renforcer les liens entre les diplômés, de favoriser l'entraide et de subvenir aux besoins de leurs veuves et orphelins ${ }^{1}$. Cette première tentative n'ira pas très loin. En outre, conçue essentiellement comme une amicale, elle ne s'étend pas aux instituteurs issus d'autres écoles qui, de leur côté, constituent des groupements similaires.

3 Il en est ainsi, par exemple, des diplômés de l'École de langue arabe (Dâr al-'ulûm, fondée en 1872), qui constituent en 1906 un Club (Nâdi Dâr al-'ulûm) présidé successivement par Hifni Nassef, Myhammad 'Atif Barakât et 'Abd al-Rahim Ahmad ce dernier est alors recteur de l'école ${ }^{2}$. Nâdi Dâr al-'ulûm porte bien son nom : il relève d'un club littéraire et d'une instance de socialisation entre élèves issus d'une même école, ayant une identité collective à affirmer et une nouvelle légitimité à construire. 
Participant à l'essor de la vie littéraire et de la production pédagogique, Nâdi Dâr al-'ulûm publie une revue à partir de $1909^{3}$.

4 Le développement, à partir de 1904, des écoles normales d'instituteurs (Ma'âhid mu'allimîn awwaliyya) favorisera la création d'associations professionnelles. Ainsi sera créé en 1910, à Qaliubiyya, un niqâba (littéralement, « syndicat » ; mais cette appellation recouvre de multiples formes et prise au pied de la lettre, elle peut être trompeuse) des instituteurs de l'enseignement élémentaire (awwali) ${ }^{4}$, et trois autres structures équivalentes au Caire et à Bunayra ${ }^{5}$. Celles-ci se donnent pour but explicite la défense des intérêts des enseignants issus des écoles de mu'allimîn awwaliyya.

\section{De l'amicale aux structures syndicales multiples : 1919-1950}

5 Tant le mouvement national de 1919 que les différenciations internes à la profession et propres à la nature des structures scolaires vont conduire à l'affirmation de multiples initiatives syndicales et à la diversification des formes de la conscience nationale et sociale parmi les enseignants, qui sont loin de constituer une catégorie socioprofessionnelle homogène.

6 Fondé en 1920, le premier «syndicat " proprement dit est présidé successivement par 'Ali bek 'Umar, Muhammad 'Atif Barakât, wakîl de l'instruction publique jusqu'à sa mort, auquel succédera le chaykh 'Atif al-Fiqqi. Tous sont issus de Dâr al-'ulûm. Ce syndicat publie une revue, Sahîfat al-mu'allimîn, qui se donne pour fonction de traiter des problèmes des enseignants et de l'instruction ${ }^{6}$. Cependant la chute du Wizârat alcha'b en 1924 et les contraintes imposées aux libertés politiques, ajoutées aux limites propres à la profession, vont conduire à l'affaiblissement progressif du syndicat puis à sa dissolution. En effet, la diversité au sein de la profession est telle qu'il est difficile à un seul regroupement de s'imposer comme le porte-parole de catégories d'enseignants de plus en plus diversifiées et dont les intérêts sont en compétition. Ces différences dépendent à la fois de leur formation, de la nature et du niveau des écoles dans lesquelles ils travaillent - écoles ahliyya (privées), amiriyya (gouvernementales) et étrangères - ainsi que des autorités dont relèvent ces dernières. Ainsi des différences notoires de statut et de salaire existent-elles entre les diplômés de Mu'allimîn 'ulya, de Dâr al-'ulûm et de Mu'allimîn awwaliyya, outre les différences de statut et de rémunération entre enseignants des écoles amiriyya, ahliyya et étrangères. Face à la multiplicité des statuts et des revendications, l'ensemble des instituteurs se détourne du syndicat et le conseil d'administration présente sa démission en janvier $1925^{7}$.

Le résultat immédiat de cet échec est de faire prévaloir à nouveau la logique associative particulariste sur la logique syndicale et revendicative. La fondation d'une association des instituteurs en $1925^{8}$ se situe dans cette perspective. Elle est constituée à l'initiative des secteurs les plus favorisés socialement et les plus «modernes» (ou présumés tels) de la profession, les diplômés de Mu'allimîn 'uliya. Muhammad Labib al-Kirdâni, recteur de l'Ecole khédiviale, est élu président et Isma'îl al-Qabbâni secrétaire ${ }^{9}$. Limitée aux diplômés de Mu'allimîn 'uliya, cette association assurera des activités à caractère pédagogique, des conférences et des débats et se gardera de toute logique revendicative ${ }^{10}$. 


\section{Les mal-aimés de l'enseignement ilzâmi}

8 C'est dans le secteur le plus défavorisé et dominé de la profession, celui des instituteurs de l'enseignement obligatoire (ilzâmi) que cette logique se manifeste : à la faveur de la Constitution de 1922, dont un des articles souligne l'importance de l'enseignement élémentaire à caractère obligatoire, un mouvement se constitue parmi les instituteurs du secteur ilzâmi. Dès 1919 sont formés des «syndicats" régionaux dont les plus célèbres sont ceux du Caire (présidé par chaykh Musaylhi Ibrahim), de Gizâ (Muhammad effendi 'Eid), de Qena (chaykh Ramadan Yusuf), de Buhayra (chaykh Muhammad 'Isa Musa), de Gharbiyya (Muhammad al-Guhari Amir), enfin d'Alexandrie (Mahmud effendi Sallam). Ces syndicats s'attaquent directement à la question de l'amélioration du statut des instituteurs du secteur ilzâmi et entreprennent des démarches à cet effet auprès des autorités ${ }^{11}$. En 1922 et 1924, des délégations d'instituteurs de ce secteur rencontrent le ministre de l'Intérieur, le wakîl al-ma'ârif et Sa'd Zaghlûl sans que ces diverses démarches aboutissent à un changement significatif ${ }^{12}$.

9 En 1928 est lancé un appel pour la formation d'une fédération des syndicats d'instituteurs de l'enseignement obligatoire, et une assemblée générale se tient au Caire en février $1929^{13}$. Le gouvernement, tentera pour sa part de contrer ce mouvement. Cependant la fédération se constitue sous la présidence du chaykh Ramadan Yusuf, installe son siège rue Muhammad 'Ali et publie une revue mensuelle des enseignants du secteur ilzâmi ${ }^{14}$.

Au cours des six années suivantes (1929-1935), cette fédération va réussir à porter sur la scène politique les problèmes de l'enseignement et des instituteurs du secteur ilzâmi. Assumant une fonction quasi-politique, organisant réunions, pétitions et publiant dans la presse, la fédération défend sa cause face à des classes dominantes qui, en dépit de la mention de l'enseignement dans la Constitution de 1922, n'en ont pas moins une attitude ambiguë face à ce type d'enseignement, destiné aux «fils de pauvres » et constituant par là même, selon ces classes, un danger social imminent. D'où la négligence à laquelle sont voués et cet enseignement et la situation de ceux qui le dispensent ${ }^{15}$. Leurs salaires ne dépassent pas en effet 4 livres (le nazir en touche 5) et ils $n$ 'ont pas droit aux augmentations annuelles ${ }^{16}$. A ces salaires correspondent 48 heures de cours par semaine, soit 8 par jour, auxquelles s'ajoutent la préparation des cours, la correction des copies et le maintien de la discipline. Le tout s'inscrivant dans des rapports hiérarchiques violents entre les hauts responsables et les instituteurs ${ }^{17}$.

11 En 1930, la fédération obtient gain de cause auprès du Parlement, qui recommande l'amélioration des salaires et des conditions de travail ${ }^{18}$. Cependant, les changements politiques et économiques de l'époque portent au pouvoir, en juin, le gouvernement d'Isma'îl Sidqi qui, d'une part, exprime les volontés du Palais et les intérêts de la haute bourgeoisie et, d'autre part, oppose un refus catégorique aux revendications des instituteurs, dans la mesure où il ne voit pas d'un bon oil la généralisation de l'enseignement ilzâmi.

12 Chaykh Ramadan Yusuf poursuit pour sa part une campagne largement répercutée dans l'opinion à propos des salaires. Requêtes et pétitions sont bientôt soutenues par les grandes signatures de l'époque et les personnalités sensibilisées aux questions de l'éducation : «La profession de l'instituteur ilzâmi n'est pas moins importante que celle d'un professeur d'université ", écrit notamment Taha Hussein ${ }^{19}$. Aqqâd ajoute pour sa 
part que « quiconque accepte de commencer sa carrière à 4 livres et de l'achever à 6 ne peut être que de vile origine, et nul n'accepterait de lui confier ses enfants $»^{20}$. Quant à Gohari 'Amir, il soutient que l'application de la loi de l'offre et de la demande aux enseignants ilzâmi par le gouvernement, qui les traite ainsi comme des marchandises, contredit les principes d'un gouvernement censé respecter l'équité : « La loi de l'offre et de la demande spécule sur les besoins des nécessiteux et les prive de leurs droits. $»^{21}$ Ibrahîm Yusuf enfin, inspecteur de l'instruction, explique la baisse du niveau de l'enseignement ilzâmi par l'esprit de révolte qui sévit dans ce secteur en raison de la médiocrité des salaires ${ }^{22}$.

13 Le gouvernement d'Isma'îl Sidqi n'en reste pas moins hostile à ces demandes. Le coup d'État anti-constitutionnel de 1930 - dissolution du Parlement, suspension de la Constitution de 1923 et promulgation de celle de 1930 - a pour effet de reconstituer un front national dont les revendications sont axées sur le retour à la vie parlementaire et à la Constitution de 1923. Les instituteurs du secteur ilzâmi se retrouvent tout naturellement dans ce mouvement. Leur statut social de diplômés restés proches de leurs origines populaires fera d'eux d'importants porte-parole de l'opposition wafdiste, notamment en milieu rural. Aussi ces prises de positions politiques leur vaudront-elles l'hostilité des grands propriétaires terriens, proches des gouvernements de minorités.

La lutte qui oppose la fédération à l'État entre 1930 et 1935 se manifeste sous plusieurs formes. Face au refus du gouvernement d'augmenter leur salaire ${ }^{23}$, les instituteurs réitèrent leur démarche auprès du Parlement. Celui-ci leur réserve, en 1933, une campagne d'une extrême violence, en représailles à leur hostilité envers le gouvernement durant les élections. Mahmud bey Zaqi, wakîl du Parlement, est en particulier à l'origine de la campagne de dénigrement qui les vise : leur compétence professionnelle est mise en cause, leurs revendications salariales perçues comme injustifiées. Cette cabale suscite une révolte parmi les instituteurs, soutenus, une fois de plus, par l'opinion et par la presse ${ }^{24}$. C'est de la part du ministre de l'Instruction, Hilmi pacha 'Isa, auprès duquel ils espéraient trouver un soutien, qu'ils vont subir le revers le plus cinglant: leurs doléances s'apparentent pour lui à une contestation de l'ordre, qu'il envisage de réduire en tentant de dissoudre la fédération. Les leaders de cette dernière sont persécutés, soumis à de multiples abus administratifs ${ }^{25}$ : son président Ramadan Yusuf est muté dans les oasis, et ses deux wakîl, Gohari 'Amir et 'Isa Musa, sont envoyés, à Qena et Aswan respectivement puis sont dégradés, passant du statut de nazir à celui d'instituteur ${ }^{26}$; leurs salaires sont dévalués en conséquence. Ce que le gouvernement vise, en fait, est essentiellement le capital symbolique et l'écoute dont jouit cette catégorie d'enseignants dans les milieux ruraux; il s'agit de neutraliser leur impact politique sur l'opinion et de réduire leur statut social de diplômés, qu'ils opposent aux grands propriétaires terriens.

15 La question de la bataille autour du costume des enseignants, qui rend compte de manière éloquente des enjeux de l'époque, va fournir au pouvoir une occasion parmi d'autres de battre en brèche ces aspirations. L'affaire éclate au moment où les instituteurs tentent de troquer le costume azharien contre l'habit civil (dit " européen ») et cherchent à se débarrasser du titre de chaykh pour devenir des effendi, à l'instar du reste des diplômés; double démarche qui vise à rehausser leur statut social dans les campagnes et à les faire apparaître enfin comme partie intégrante des secteurs modernisés de la société. Or, non seulement le gouvernement oppose un nouveau refus à cette revendication d'appartenance, mais encore, le costume azharien est imposé aux 
instituteurs pour les tenir à l'écart du statut social auquel ils aspirent. Un vaste débat $s^{\prime}$ ensuit ${ }^{27}$, le pouvoir campant sur ses positions face aux instituteurs, qui vont jusqu'à adresser leur plainte au Palais ${ }^{28}$. Le costume relève de la liberté individuelle, disent-ils, s'il n'y a pas de contrainte en matière de religion, comment pourrait-il y en avoir en matière vestimentaire ? ${ }^{29}$

L'enjeu de cette bataille du costume est bien celui de la prétention des instituteurs à la modernisation et à la prise en charge de nouveaux rôles et médiations sociales, prétention que leur dénient dirigeants et élites. Des peines de prison et de renvoi sont prévues pour les rebelles ${ }^{30}$, et le directeur de l'enseignement à Daqahliyya donne l'ordre aux 'umda et chefs de villages d'arrêter tout récalcitrant; dans cette même région, cinq instituteurs sont radiés ${ }^{31}$.

Incapable de tenir tête au gouvernement, la fédération des instituteurs du secteur ilzâmi s'affaiblit peu à peu. Avec la chute du gouvernement de Sidqi et la nomination de Nagib al-Hilâli à la tête du Ministère de l'Instruction en novembre 1934, elle est davantage prête à accepter des compromis avec l'État. Elle a éloigné certains de ses membres les plus intransigeants en échange de l'acceptation, par Hilâli, de certaines de ses revendications. Ramadan Yusuf, notamment, se voit écarté de la présidence de la fédération et remplacé par Gohari 'Amir ${ }^{32}$, plus proche du pouvoir et qui maintiendra le contrôle de la fédération jusqu'en 1953, vidant le mouvement de son contenu syndical et revendicatif.

La condition des instituteurs du secteur ilzâmi va toutefois connaitre une certaine amélioration après la nomination de Taha Hussein à la tête de l'Instruction dans le dernier gouvernement du Wafd (1950-51). A la faveur du slogan 'Al-'ilm ka-l-ma' wa-lhawa' ("le savoir est accessible comme l'eau et l'air »), l'enseignement obligatoire est perçu comme fondement d'une édification sociale plus démocratique. Défavorisés du système obligatoire gratuit et élèves des écoles primaires se retrouvent dans un système unifié et les salaires des instituteurs sont augmentés. Taha Hussein ayant à nouveau suscité des espoirs parmi eux ${ }^{33}$, la fédération reprend vie, reconstitue ses structures et relance la publication de sa revue ${ }^{34}$. Avec la constitution, en 1951, d'un Ordre national regroupant tous les enseignants, les instituteurs de l'enseignement obligatoire (désormais primaire) rejoignent la structure unitaire. Cela ne va pas entraîner pour autant la disparition de leurs propres formes de regroupement; la fédération se maintient sous le nom de "Fédération des instituteurs du primaire " $^{35}$ puis, à partir de 1958, «Association centrale de l'enseignement primaire ${ }^{36}$. Sa revue a perduré37, d'abord présidée par al-Gohari 'Amir auquel a succédé 'Awad Allah 'Uthman's8.

\section{«Clubs » et sociétés savantes}

19 C'est toujours à la suite de la dissolution, en 1925, de l'Ordre des enseignants que les diplômés de Dâr al-'ulûm tentent de mettre en place une structure qui leur soit propre. Celle-ci leur semblait d'autant plus nécessaire qu'ils étaient soumis à une exploitation croissante de la part des écoles privées. En 1932 est donc fondé un Groupement de l'enseignement libre (privé) de Dâr al-'ulûm, qui entrera en conflit tant avec le ministère qu'avec les propriétaires des écoles privées mais réussira à obtenir quelques améliorations ${ }^{39}$. 
20 Plus culturelle que revendicative, la Société de Dâr al-'ulûm est constituée en 1933 et publie une nouvelle revue. En 1935, elle se transforme en un club présidé par le chaykh Abu-l-Fath al-Fiqi, auquel succède en 1936 et pour dix ans Muhammad Nagib Hatata. Par la suite, Groupement de l'enseignement privé et Société fusionnent sous la présidence de Sa'd al-Labban ${ }^{40}$.

21 Fondée en 1939, Rabitat al-tarbiyya al-hadîtha, la Ligue de l'enseignement moderne ${ }^{41}$, semble correspondre à une nouvelle logique et à de nouveaux acteurs. L'enjeu est bien celui de la valorisation d'un savoir nouveau, de rôles sociaux inédits dont il est le corollaire et enfin, de la double apparition de l'expertise et de l'expérimentation dans le domaine de l'éducation. Expertise et expérimentation dont les conditions intellectuelles et matérielles de mise en œuvre sont étroitement liées à l'affirmation sociale de ceux qui seront amenés à exercer ces fonctions. La Rabita est en effet constituée de catégories d'enseignants «modernes » et présidée par Ahmad 'Abd alSalam al-Kirdâni, doyen de Ma'had al-tarbiyya li-l-mu'allimin. Ses orientations sont d'abord pédagogiques: elle diffuse auprès des enseignants égyptiens les nouvelles méthodes pédagogiques européennes, et mène des recherches et des études de terrain visant à mieux cerner la spécificité de la situation éducative en Égypte et ce qu'elle requiert d'innovation pédagogique ${ }^{42}$.

Ces aspects se renforcent avec la création, en 1943, de la Ligue des diplômés des instituts pédagogiques, qui se situe dans la même logique ${ }^{43}$ : «diffuser les principes d'une pédagogie moderne, favoriser son application dans les écoles et renforcer les liens scientifiques et humains entre ses membres ». Présidée à l'époque par Isma'îl alQabbâni, elle organise des conférences, des camps de formation, des colloques scientifiques. Elle publie en outre Majallat al-tarbiyya (Revue de pédagogie ou Revue d'éducation, le terme de tarbiyya rendant compte des deux notions) exclusivement consacrée aux recherches dans ce domaine ${ }^{44}$.

Du côté des enseignantes - mais dans une perspective plus orientée vers la spécificité de leurs conditions de travail - se dessine un mouvement parallèle. En 1945, Asma Fahmi, wakîla de l'Institut de formation des institutrices, fonde une Ligue des diplômées des instituts de pédagogie. Avancée d'autant plus importante dans le mouvement des institutrices que ces dernières étaient en effet soumises à des conditions de travail particulières. Entre 1925 et 1940, la question du droit au mariage des institutrices en service - dénié par le ministère - fait l'objet d'un débat public auquel participent les féministes. Sous les pressions de la Ligue, le ministère cède du terrain et les institutrices sont réparties en trois catégories en fonction du diplôme : les diplômées des instituts supérieurs de pédagogie, qui sont autorisées à se marier; celles issues de l'Ecole des institutrices saniyya, qui le sont également mais sous réserve de l'accord préalable du ministère. Quant aux institutrices issues des instituts awwali, elles ne peuvent se marier sous peine d'être radiées ${ }^{45}$. Ces distinctions en fonction du diplôme - qui reprennent de bout en bout, dans une version féminine, la hiérarchie au sein des enseignants - suscitent de nombreuses et violentes critiques face auxquelles le ministère cède ${ }^{46}$. La Ligue des institutrices finira par fusionner avec celle des diplômés des facultés de pédagogie en $1953^{47}$.

24 A ces formes syndicales de regroupement s'ajoutent, sur une période allant de 1919 à 1951, d'autres structures et instances qui éclairent la spécificité des revendications et des statuts, elle-même reflet de la multiplicité des intérêts en jeu, des acteurs, des niveaux et des filières de l'enseignement. Citons le Syndicat des écoles ahliyya, la 
Fédération des diplômés d'al-Azhar, la Fédération générale de l'enseignement libre, le Club de la faculté de langue arabe, l'Union des diplômés des écoles secondaires industrielles, la Fédération des Beaux-Arts, la Ligue des enseignants des écoles industrielles, celle des diplômés des instituts agricoles, des enseignants de langue française, des diplômées des cours externes, la Fédération des instituts de formation des instituteurs du primaire, l'Union des enseignants de dessin ou la Ligue des enseignants d'université... ${ }^{48}$

\section{Vers un mouvement unifié des instituteurs : 1951-1981}

Si, à la multiplicité des formes de regroupement, correspondait celle des statuts, il faut croire également que l'État, compte tenu des effectifs enseignants, voyait d'un mauvais œil la perspective d'un mouvement unifié et susceptible de constituer une véritable force ${ }^{49}$. C'est à la faveur de l'arrivée, à la tête du Ministère de l'Enseignement, de Taha Hussein, durant le dernier cabinet du Wafd (1950-1951), que se met en place la structure syndicale unifiée longtemps recherchée. La loi 319 de 1951 stipule la création d'un ordre regroupant tous les personnels enseignants, à l'exception de ceux des universités et d'al-Azhar ${ }^{50}$. Cette loi ne prendra pas effet immédiatement en raison des troubles politiques qui agitent l'Égypte en 1951-52. L'ordre ne verra le jour qu'en 1955, après un amendement de la loi.

La première assemblée générale, le 18 février 1955, se solde par l'élection du lieutenant Kamal al-Din Hussein, membre des Officiers libres et ministre de l'Enseignement, à la tête du Syndicat des instituteurs ${ }^{51}$. Ce moment inaugure une nouvelle période du mouvement syndical des enseignants. Paradoxalement, son unification aura eu pour effet, non de le transformer en groupe de pression, mais de le rendre plus vulnérable face à l'administration - représentée ici par le Ministère de l'Enseignement - et de faciliter la mainmise de l'État sur l'une des professions aux effectifs les plus nombreux, à l'impact social le plus important - quoique diffus - mais au poids politique et à la capacité de défense particulièrement faibles de ses intérêts propres.

Kamal al-Din Hussein occupe les fonctions de ministre de l'Enseignement et de président du syndicat pendant dix ans, durant lesquels ce dernier se transforme en structure quasi gouvernementale, dépendante du pouvoir exécutif, incapable de faire face aux problèmes de la profession et mise au service d'un réseau de clientèle ${ }^{52}$. Parallèlement, les salaires des enseignants restent inférieurs à ceux de l'ensemble des professions de la fonction publique. En 1964, un mouvement d'enseignants se constitue, qui revendique une amélioration des salaires. Il est réprimé par la violence et ses principaux instigateurs exclus de la profession ${ }^{53}$. L'opération est menée par al-Sayyid Yusuf, ministre de l'Enseignement, qui présidera le syndicat jusqu'en 1971. Durant cette période, le syndicat se voit réduit à un organe de soutien politique de l'État et une annexe du ministère : peu à peu, les enseignants s'en désintéressent ${ }^{54}$.

Nommé ministre de l'Enseignement en 1968, Muhammad Hilmi Murad tente de modifier la loi du syndicat en vue d'une réforme globale, tentative mise en échec par le président du syndicat qui, lui-même, en sera écarté lors du «mouvement de redressement " entrepris par le président Sadate le 15 mai $1971^{55}$. Il est peu probable que le pluralisme limité ait eu, sur le syndicat des enseignants, un effet autre que celui de renforcer le contrôle de l'appareil d'État. En juin 1971, Muhammad Mahmud 
Radwan, vice-ministre de l'Enseignement, est placé à la tête du syndicat, où il demeurera jusqu'en avril $1981^{56}$. Durant cette période, les services offerts par le syndicat à ses membres ne cessent de se dégrader et la corruption continue d'être le trait marquant d'une structure dont les responsables trouvent appui auprès du pouvoir, au risque de voir le syndicat se réduire au groupe qui en tire directement des rentes.

\section{Le mouvement syndical aujourd'hui}

Entre 1981 et 1991, deux personnalités politiques se partagent la présidence du Syndicat des enseignants. En avril 1981, Mansur Hussein, vice-ministre de l'Enseignement, président de la commission du PND pour l'enseignement puis ministre en 1985-86 - et par la suite membre du Conseil consultatif -, occupe les fonctions de président de l'Ordre pour deux mandats successifs (1981-85 et 1985-89) ${ }^{57}$. Il reconduit ici ce qu'il faudrait considérer comme une "tradition": le cumul des fonctions de ministre de l'Enseignement et de président du syndicat ${ }^{58}$.

Lorsqu'il devient ministre de l'Enseignement, la presse rend compte d'une anecdote révélatrice du fonctionnement de l'Ordre des enseignants : son principal concurrent aux élections du syndicat, un inspecteur de langue arabe qui l'a accusé de vol et de fraude durant la campagne électorale, est le premier à organiser la cérémonie d'investiture du nouveau ministre ${ }^{59}$. Plus tard, quand Mansur Hussein sera exclu du ministère, le syndicat du Caire évoquera, dans un communiqué, un «scandale financier » où il semble avoir été impliqué ${ }^{60}$.

31 N'étant guère rompus, de par leur formation, à l'exercice de la démocratie, les enseignants ont peu à peu abandonné le syndicat aux mains d'une minorité qui a monopolisé le travail syndical et l'a utilisé pour ses intérêts privés. Le syndicat se retrouve isolé par rapport aux structures équivalentes, tant dans le monde arabe que sur le plan international, et son fonctionnement s'est détérioré à un point tel que c'est maintenant du côté de la presse pro-gouvemementale que viennent les critiques ${ }^{61}$. Par ailleurs, le système de retraite complémentaire et de services offerts au corps enseignant reste inférieur à celui dont bénéficient les autres professions ${ }^{62}$.

Cet état de fait prévaut encore actuellement. Depuis 1989 a succédé à Mansur Hussein, à la tête du syndicat, Mustafa Kamal Hilmi, ministre de l'Enseignement entre 1974 et 1984 et détenteur de la fonction politique importante de président du Conseil consultatif ; tel semble être le curriculum idéal pour un président de syndicat ${ }^{63}$. Le début de son mandat s'est accompagné d'une nette augmentation des cotisations pour combler le déficit budgétaire et améliorer les services et les retraites complémentaires ${ }^{64}$. Cependant, en dépit de ces mesures, le syndicat n'a pas connu de transformations radicales. Absorbé par ses fonctions politiques, son président est accusé par la presse de négliger les intérêts de plus d'un demi million d'enseignants ${ }^{65}$. Le conseil du syndicat ayant préféré ignorer pour sa part les signes d'une mobilisation contestataire dans certaines branches de province ${ }^{66}$, son président, lors de la $34^{\mathrm{e}}$ assemblée générale, a été accusé de faire le jeu du gouvernement aux dépens des revendications des instituteurs ${ }^{67}$. Il s'en est suivi, de la part du premier ministre et du ministre des Finances, des promesses d'augmentation de salaire qui avaient surtout pour objectif d'apaiser les esprits ${ }^{68}$. Ainsi Mustafa Kamal Hilmi a-t-il été réélu en avril 1993. L'Ordre des enseignants redevenait 
ce que le leader syndicaliste ouvrier 'Atiyya al-Sirafi décrit comme "une immense pyramide bourrée de momies ${ }^{69}$.

\section{Aspects de la crise}

II s'agit là, en définitive, d'une des manifestations de la crise que connaissent certaines sociétés du tiers-monde, prises entre des déclarations d'intention démocratique et des structures autoritaires. Dans le cas du mouvement des enseignants, elle se manifeste tout d'abord, et de la manière la plus éloquente, à travers le cumul des fonctions de ministre et de président du syndicat, phénomène ancien, inscrit dans l'histoire même $\mathrm{du}$ mouvement. Pour y faire face, certains enseignants appellent à l'élection directe du président du syndicat par l'assemblée générale. Ce serait également une manière de mettre fin au contrôle exercé par les supérieurs administratifs sur l'activité syndicale ${ }^{70}$.

La professionnalisalisation de l'activité syndicale constitue un autre aspect, non moins significatif, de la crise. Le vice-président actuel du syndicat (1993) était membre du premier conseil, formé en 1955. Pendant 38 ans, il a, sous une forme ou une autre, maintenu une présence. Bien que la loi régissant le syndicat stipule qu'un membre ne peut être élu pour plus de deux mandats consécutifs et pour une durée maximale de huit ans, les professionnels du syndicalisme sont parvenus à contourner cette contrainte.

La mainmise de l'exécutif ne se réduit pas seulement au cumul de fonctions évoqué plus haut; on la retrouve à tous les échelons de la structure syndicale, miroir des structures administratives ; par exemple, le directeur de l'enseignement au niveau du gouvernorat est également président de la branche de province du syndicat. La présence systématique de hauts responsables au sein des structures syndicales fait de l'adoption des options gouvernementales un automatisme, et de la mainmise de l'administration sur le syndicat une "tradition", syndicale et politique à la fois ${ }^{71}$. Il s'ensuit que la représentation au sein du syndicat, loin de s'ouvrir aux instituteurs de base, demeure exclusivement limitée aux supérieurs hiérarchiques ${ }^{72}$. L'activité syndicale est menée dans un esprit de fonctionnariat. Quant au conseil du syndicat, il soutient globalement les options du pouvoir ${ }^{73}$ et, en contrepartie, est assuré de sa protection contre les critiques émises par la base.

L'absence de démocratie s'inscrit également dans les dispositions formelles de fonctionnement $\mathrm{du}$ syndicat: les élections à plusieurs échelons en constituent un aspect représentatif. Une profession de plus d'un demi million d'individus est représentée par une assemblée générale de 1.500 membres qui élisent le président et le conseil d'administration. On se retrouve ainsi face à un système d'élections à trois échelons : comité local, branche syndicale du gouvernorat et enfin syndicat général au Caire. A cela s'ajoute le fait que deux catégories de personnes détiennent traditionnellement les sièges des conseils d'administration: d'une part, les hauts fonctionnaires, de l'autre, les instituteurs de base soumis à des pressions hiérarchiques et administratives telles qu'ils se trouvent acculés à opter pour les choix de la direction. Le regroupement dans une même instance représentative et élue n'a pas eu pour effet de réduire les écarts entre ces deux groupes. Bien au contraire, ils sont confirmés dans la pratique quotidienne, où l'on voit se dessiner deux clans: celui des supérieurs hiérarchiques et administratifs, qui prennent les décisions, et celui des "clients", instituteurs de base, qui les exécutent. 
Le syndicat des enseignants se caractérise enfin par une dégradation du niveau des services et avantages offerts à la profession. Ses fonds sont souvent investis dans des projets non rentables, ce qui a poussé certains enseignants à réclamer le contrôle de la Cour des comptes. Le syndicat n'est intervenu de façon efficace ni pour obtenir une augmentation significative des salaires, qui restent inférieurs à ceux de la fonction publique, ni pour revaloriser une profession dont l'image de marque ne cesse de se dégrader. Une enquête réalisée auprès des enseignants montre que 93 \% d'entre eux considèrent le syndicat comme une structure dénuée d'impact ${ }^{74}$. Ajoutons que si, parmi les préoccupations d'un syndicat, figure en principe le souci de l'éthique de la profession, le fait est que dans ce domaine, le syndicat des enseignants a plutôt renforcé leur passivité ${ }^{75}$. Leur mouvement syndical est d'autant plus vulnérable que leurs besoins économiques sont pressants et leurs revenus limités. Ils appartiennent en effet, pour la plupart, aux classes défavorisées qui n'ont que leur salaire pour moyen de subsistance. La multiplication des cours particuliers, qui leur permettent de réaliser des compléments de revenus non négligeables, a été avalisée par le syndicat. Reste enfin à noter que l'ensemble de ces caractéristiques - absence de démocratie et conditions économiques précaires - constitue un terrain favorable à l'émergence de courants religieux extrémistes dans les milieux enseignants égyptiens. Aujourd'hui, le Ministère de l'Enseignement appelle à se mobiliser contre ces courants. Cela ne peut réussir que sous réserve de transformations socio-économiques radicales, et à condition de libérer le syndicat des enseignants des liens qui entravent son action.

\section{NOTES}

1. Gam'iyyat al mu'allimîn, Al-Kitâb al-zahabi li madrasat al-mu'allimîn al-'uliya (Livre d'or de Madrasat al-mu'allimîn al-'uliya), Le Caire, 1937, p. 248.

2. 'Abd Al-Gawwad M., Taqwim Dâr al-'ulûm (Annuaire de Dâr al-'ulùm). Dâr al-ma'ârif, LeCaire, p. 705.

3. Sahîfat Nâdi Dâr. al-'ulûm, avril 1909.

4. 'Izz Al-Din A., Târikh al-tabaqa al-'âmila (Histoire du mouvement ouvrier). Le Caire, 1970. p. 97.

5. Abu-l-As'ad M-, Târikh at-ta'lîm fi Misr 1882-1922 (Histoire de l'enseignement en Égypte), Thèse de magistère non publiée, faculté des lettres, Université de 'Ayn Chams, 1976, p. 314.

6. Sahîfat al-mu'allimîn, février 1923.

7. Sahîfat al-mu'allimîn, février 1925.

8. Majallat al-tarbiyya al-hadîtha, mars 1928.

9. Sahîfat al-mu'allimîn, février 1925.

10. Majallat al-tarbiyya al-hadîtha, mars 1928.

11. Sahîfat al-mu'allimîn al-ilzâmi, mai 1934.

12. Al-Ra'id, mars 1971.

13. Sahîfat al-ta'lîm al-ilzâmi, mai 1934.

14. Niqâbat al-mihan al-ta 'limiyya, Al-khâlidûn (Les immortels). Le Caire, 1986, p. 67.

15. Abu-l-As'ad M., Majjâniyyai al-ta'lîm (La gratuité de l'enseignement), Le Caire. 1989, p. 56.

16. Niqâbat al-mihan al-ta 'limiyya, op. cit., p. 70. 
17. Sahîfat al-ta'lîm al-ilzâmi, avril 1935.

18. Al-Ra'id, février 1971.

19. Sahîfat al-ta'lîm al-ilzâmi, juillet 1934 .

20. Niqâbat al-mihan al-ta 'limiyya, op. cil., p. 75.

21. Sahîfat al-ta'lîm al-ilzâmi, mars 1935.

22. Idem, juillet 1934.

23. Idem, octobre 1933.

24. Idem, décembre 1934.

25. Al-Ra 'id, février 1972.

26. Sahîfat al-ta'lîm al-ilzâmi, décembre 1933.

27. Matar 'A. F., Al-ta'lîm wa-l-mula'atilûn (L'enseignement et les chômeurs), Alexandrie,1939, p.

82.

28. Sahîfat al-ta'lîm al-ilzâmi, novembre 1933.

29. Idem, décembre 1934.

30. Idem. novembre 1933.

31. Idem, juin 1934.

32. Idem, septembre 1935.

33. Al-mu'allim al-awwali, septembre 1951.

34. Idem, octobre 1951.

35. Idem, janvier 1953.

36. Cette association garde toujours un siège, rue Najib al-Rihâni au Caire.

37. Al-mu'allim al-awwali, décembre 1962.

38. Al-Ra'id, mai 1970.

39. 'Abd Al-Gawwad, op. cit, p. 511.

40. Ibid., p, 522 .

41. Rabitat al-tarbiyya al-hadîtha, Al-madrasa al-awwaliyya al-rifiyya (L'école élémentaire rurale), Le Caire, 1940.

42. Présidée aujourd'hui par Sa'id Isma'îl 'Ali.

43. Présidée aujourd'hui par Mahmud 'Abd al-'Aziz.

44. Rabitat kharijji kuliyyal al-tarbiyya, Mu'tamar asalib al-tarbiyya al-haditha (Colloque sur les méthodes d'éducation moderne), Le Caire, 1945.

45. Salem L. M., Al-mar'a al-misriyya wa-l-taghayur al-ijtimấi (La femme égyptienne et le changement social), Le Caire, 1984. p. 112.

46. Musa M., Hayati bi-qalami (Autobiographie), s. d., p. 329.

47. Sahîfat al-tarbiyya, novembre 1968.

48. Al-Ahrâm, 2 février1955.

49. Nasr M. I., Qânûn niqâbat al-mu'allimîn (Loi de l'ordre des enseignants), Le Caire, 1952. p. 4.

50. Abu-l-As'ad M., Tatawwur al-haraka al-niqâbiyya li-l-mu'allimîn al-misriyyîn 1951-1981 (Evolution du mouvement syndical des instituteurs égyptiens), Le Caire, 1992, p. 18

51. Al-mu'allim al-awwali, mars 1950.

52. Abu-l-As'ad, Tatawwur, op. cit., p. 40.

53. Sahifat al-tarbiyya. avril 1976.

54. Abu l-As'ad, Tatawwur, op. cit., p. 64.

55. Al-Ahrâm, 6 juin1971.

56. Abu l-As'ad, Tatawwur, op. cit.. p. 89.

57. Al-Gumhuriyya, 20 avril1981.

58. Al-Ahrâm al-iqtisâdi, 1er mai 1989.

59. Al-Ahâli. 18 septembre 1985.

60. Selon Al-Ahâli 12 novembre 1986, la campagne de « modernisation de l'enseignement » lancée par Mansur Hussein aurait essentiellement consisté en l'achat, à une société privée, de 12.500 
ordinateurs destinés à équiper des écoles menaçant de s'écrouler, cela au moment même où le fils du ministre était recruté au sein de ladite société.

61. Al-Ahrâm al-iqtisâdi, 31 octobre 1988.

62. Ibid., $1^{\mathrm{er}}$ février1989.

63. Ibid., 24 octobre 1988.

64. Al-Gumhuriyya, 27 avril 1992.

65. Al-Ahrâm al-iqtisâdi, 4 juin 1990.

66. Al-Cha'b, 28 avril 1992.

67. Misr al-Fatah, 27 avril 1992.

68. Al-Cha'b, 28 avril 1992.

69. Al-Sirafi 'A., 'Askarat al-hayat al-'ûmmâliyya wa-l-niqâbiyya fi Misr (La militarisation de l'activité ouvrière et syndicale en Égypte), Le Caire, 1982, p. 57.

70. Al-Taira, avril 1976.

71. Bayumi K. H., Dirâsa mûqârana li-l-dawr al-tarbawi li-niqâbât al-mihan af-ta'lîmiyya (Etude comparée du rôle des syndicats d'enseignants en matière pédagogique), thèse de doctoral $n . p$., Fac. de pédagogie, Univ. 'Ayn Chams, 1988, p. 82.

72. Sahîfat al-tarbiyya, juin 1976.

73. Al-Sayyid M. K., Al-mujtama' wa-l-siyassa fi Misr (Politique et société en Égypte), Le Caire, 1983, p. 87.

74. Hassan A,. Dawr niqâbat al-mihan as-ta'Iimiyya (Le rôle du syndicat des enseignants), thèse de magistère non publiée, Faculté de Jeunes filles, Université 'Ayn Chams, 1983, p. 72.

75. Ali S. I., Innahûm yûkharribûn at-talîm (On sabote l'enseignement), Le Caire. 1986, p. 83.

INDEX

Mots-clés : éducation, enseignement, histoire, professeur, syndicalisme

\section{AUTEURS}

\section{MUHAMMAD ABU-L-AS'AD}

Université de Minia 